Article

\title{
Clostridium butyricum MIYAIRI 588 Increases the Lifespan and Multiple-Stress Resistance of Caenorhabditis elegans
}

\author{
Maiko Kato ${ }^{\dagger}$, Yumi Hamazaki ${ }^{\dagger}$, Simo Sun, Yoshikazu Nishikawa * ${ }^{(D)}$ and \\ Eriko Kage-Nakadai *(D) \\ Graduate School of Human Life Science, Osaka City University, Osaka 558-8585, Japan; \\ chisukonya@yahoo.co.jp (M.K.); yumi.hamazaki123@gmail.com (Y.H.); sunsimo1986511@gmail.com (S.S.) \\ * Correspondence: nisikawa@life.osaka-cu.ac.jp (Y.N.); nakadai@life.osaka-cu.ac.jp (E.K.-N.); \\ Tel.: +81-6-6605-2900 (Y.N.); +81-6-6605-2856 (E.K.-N.) \\ + These authors equally contributed to this work.
}

Received: 26 October 2018; Accepted: 2 December 2018; Published: 5 December 2018

check for updates

\begin{abstract}
Clostridium butyricum MIYAIRI 588 (CBM 588), one of the probiotic bacterial strains used for humans and domestic animals, has been reported to exert a variety of beneficial health effects. The effect of this probiotic on lifespan, however, is unknown. In the present study, we investigated the effect of CBM 588 on lifespan and multiple-stress resistance using Caenorhabditis elegans as a model animal. When adult C. elegans were fed a standard diet of Escherichia coli OP50 or CBM 588, the lifespan of the animals fed CBM 588 was significantly longer than that of animals fed OP50. In addition, the animals fed CBM588 exhibited higher locomotion at every age tested. Moreover, the worms fed CBM 588 were more resistant to certain stressors, including infections with pathogenic bacteria, UV irradiation, and the metal stressor $\mathrm{Cu}^{2+}$. CBM 588 failed to extend the lifespan of the daf-2/insulin-like receptor, daf-16/FOXO and skn-1/Nrf2 mutants. In conclusion, CBM 588 extends the lifespan of C. elegans probably through regulation of the insulin/IGF-1 signaling (IIS) pathway and the Nrf2 transcription factor, and CBM 588 improves resistance to several stressors in C. elegans.
\end{abstract}

Keywords: Clostridium butyricum; lifespan; stress resistance; Caenorhabditis elegans

\section{Introduction}

Probiotics are defined as live microorganisms that, when administered in adequate amounts, confer health benefits to the host [1]. Clostridium butyricum, which is an anaerobic, Gram-positive, spore-forming bacillus, is one of the commercialized probiotic bacteria. C. butyricum is distributed in a variety of environments and in the gut of various animals, including humans [2]. Several studies have described the prevalence of $C$. butyricum; $44 \%$ of fecal samples collected from asymptomatic preterm neonates were positive for C. butyricum [3], and C. butyricum was identified in $10 \%$ to $20 \%$ of human fecal samples by microbial culture [4].

C. butyricum MIYAIRI 588 (CBM 588) is used as an anti-diarrheal medicine in Japan. In clinical practice, the administration of CBM 588 was shown to prevent antibiotic-associated diarrhea in children [5]. Numerous experimental studies have demonstrated the beneficial properties of this probiotic, including the induction of interleukin-10 (IL-10)-producing macrophages in an experimental mouse model of colitis [6], the promotion of regulatory T-cell generation in the intestine through transformation of growth factor- $\$$ (TGF-ß) from dendritic cells [7], the improvement of high-fat diet-induced non-alcoholic fatty liver disease in rats [8], the inhibition of the cytotoxic effect of Clostridium difficile in vitro [9], and the prevention of enterohemorrhagic Escherichia coli O157:H7 
infection [10] and gastric ulcers in mice [11]. The effect of C. butyricum on longevity, however, is unknown.

Caenorhabditis elegans, a free-living nematode that feeds on bacteria, is a powerful experimental model due to its ease of cultivation, its short and reproducible lifespan, and the abundance of genetic tools available for studying this organism [12]. Although C. elegans was originally isolated from rich soil or compost [13], where it is mostly found in the dauer stage [14,15], feeding and reproducing stages of this species have been found in microbe-rich habitats such as rotting fruit and plant matter [16,17]. Three independent studies that provided the first description of the microbiome of C. elegans [18-20], and a meta-analysis of the data [21], showing possible functions of the naturally associated bacteria; e.g., the microbes enhance $C$. elegans fitness under standard and also stressful conditions, and confer resistance to infection [19]. Specific microbes correlate with the population state of $C$. elegans and the bacterial influence is not simply nutritional [20]. In the laboratory, C. elegans is typically maintained on agar plates and fed Escherichia coli laboratory strains (e.g., OP50) [22]. We previously showed that feeding on lactic acid bacteria, including lactobacilli and bifidobacteria, extends the lifespan of worms compared with feeding on E. coli OP50 [23]. Afterwards, several lines of investigation addressed the interactions between worms and probiotic bacteria, yielding insights into mechanisms by which probiotic bacteria enhance immune responses and increase lifespan in the C. elegans host $[24,25]$. Thus, C. elegans has been an accepted model for investigating dietary manipulation with probiotics [26,27].

In the present study, we examined the influence of CBM 588 on the lifespan of C. elegans. CBM 588 was shown to extend the lifespan probably through regulation of the insulin/IGF-1 signaling (IIS) pathway and the Nrf2 transcription factor and to improve resistance to several stressors in C. elegans.

\section{Materials and Methods}

\subsection{Bacterial Strain and Culture Conditions}

Escherichia coli OP50, which is used as the standard feed for C. elegans cultivation, was grown on tryptone soya agar (Nissui Pharmaceutical, Tokyo, Japan) at $37^{\circ} \mathrm{C}$. Clostridium butyricum MIYAIRI 588 (CBM 588), which was kindly provided by Miyarisan Pharmaceutical, was cultured on GAM agar (Nissui Pharmaceutical, Japan). The Salmonella enterica serovar Enteritidis strain SE1, originally isolated from a diarrheal specimen and used as a pathogen, was grown on tryptone soya agar at $37^{\circ} \mathrm{C}$. Similarly, Staphylococcus aureus NBRC13276 was cultured on tryptone soya agar. The cultured bacteria were scraped and weighed. Aliquots (100 mg wet weight) of bacteria were suspended in $0.5 \mathrm{~mL}$ of M9

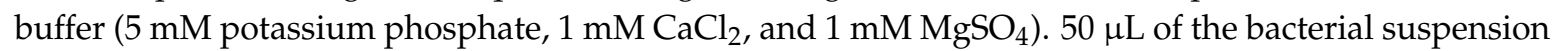
was spread onto NGM modified to be peptone free (mNGM) plates $(1.7 \%(w / v)$ agar, $50 \mathrm{mM} \mathrm{NaCl}$, $1 \mathrm{mM} \mathrm{CaCl} 2,5 \mu \mathrm{g} / \mathrm{mL}$ cholesterol, $25 \mathrm{mM} \mathrm{KH}_{2} \mathrm{PO}_{4}$, and $\left.1 \mathrm{mM} \mathrm{MgSO}_{4}\right)$ (10 mg bacteria per plate), and used in the experimental assays.

\subsection{C. elegans Strains and Culture Conditions}

The wild-type C. elegans strain Bristol N2 and the following derivative mutant strains were obtained from the Caenorhabditis Genetics Center: CB1370 daf-2(e1370), no outcross; CF1038 daf-16(mu86), outcrossed with N2 eleven times by the Kenyon lab.; and VC1772 skn-1(ok2315), outcrossed one time by the Moerman lab. These C. elegans strains were maintained using standard techniques [22]. The animals were cultured as follows: eggs were prepared from adult C. elegans by exposure to a sodium hypochlorite/sodium hydroxide solution. The egg suspension was incubated in M9 buffer for one day at $25^{\circ} \mathrm{C}$ to allow for hatching and synchronization, and the resulting suspension of synchronized L1-stage worms was centrifuged at $156 \times g$ for $1 \mathrm{~min}$. After the removal of the supernatant by aspiration, the remaining larvae were transferred onto mNGM plates covered with $10 \mathrm{mg}$ of OP50. The transferred worms were cultured at $25^{\circ} \mathrm{C}$ for two days (referred to as three-day-old animals). daf-2(e1370) animals were grown at $15^{\circ} \mathrm{C}$, the permissive temperature, until they reached the young adult stage. 


\subsection{Determination of the C. elegans Lifespan}

Lifespan assays were performed as follows: synchronized three-day-old (young adult) animals (35 animals per plate) were placed on a 5-cm mNGM plate covered with $10 \mathrm{mg}$ of OP50 alone, CBM 588 alone, or a mixture of $5 \mathrm{mg}$ each of OP50 and CBM 588, and then, the plates were incubated at $25{ }^{\circ} \mathrm{C}$. The animals were transferred daily to fresh plates for the first four days, and thereafter, they were transferred every other day. The number of live and dead animals was recorded every day. An animal was considered dead when it failed to respond to a gentle touch with a worm picker. Animals that crawled off the plate or died from internal hatching were considered lost and not included in the analysis. Each assay was carried out in duplicate (two plates). Two independent replicates were performed except for experiments using UV-killed bacteria and mutant analysis using daf-2(e1370). The data from the replicates were merged, and presented. Worm survival was calculated by the Kaplan-Meier method, and differences in survival were analyzed using the log-rank test.

The mean lifespan (MLS) was estimated using the following formula [28]:

$$
\mathrm{MLS}=\frac{1}{N} \sum_{j} \frac{x_{j}+x_{j+1}}{2} d_{j}
$$

where $d_{j}$ is the number of worms that died in the age interval $\left(x_{j}\right.$ to $x_{j+1}$ ), and $N$ is the total number of worms. The standard error (SE) of the estimated mean lifespan was calculated using the following equation:

$$
\mathrm{SE}=\sqrt{\frac{1}{N(N-1)} \sum_{j}\left(\frac{x_{j}+x_{j+1}}{2}-\mathrm{MLS}\right)^{2} d_{j}}
$$

Maximum lifespan was calculated as the mean lifespan of the longest-living $15 \%$ of the worms in each group.

\subsection{Measurement of Body Size}

Three-day-old adult worms were placed on mNGM plates covered with lawns of OP50, CBM 588, or a mixture of the two strains. The plates were incubated at $25^{\circ} \mathrm{C}$, and the body size of the live worms was measured every $24 \mathrm{~h}$ until the worms reached an age of 10 days. Images of adult nematodes were taken with an M165FC stereoscopic microscope (Leica microsystems, Wetzlar, Germany) equipped with a DFC425C camera (Leica microsystems, Germany) and analyzed using ImageJ software. The area of a worm's projection was used as an index of the body size.

\subsection{Measurement of Brood Size}

Two L4-stage hermaphrodites were transferred to an mNGM plate covered with a lawn of OP50, CBM 588, or a mixture of the two strains. The parental animals were transferred to fresh mNGM plates every $24 \mathrm{~h}$ until the end of the reproductive period. The resulting eggs were left to hatch, and the number of progeny was then determined. Two independent replicates were tested to verify reproducibility, and the merged data were statistically analyzed and presented.

\subsection{Measurement of Locomotion}

Three-day-old adult worms were placed on mNGM plates covered with lawns of OP50, CBM 588, or a mixture of the two strains. The plates were incubated at $25^{\circ} \mathrm{C}$, and the body size of live worms was measured every other day from days 7 to 23 . The motility of the worms was then examined using the scoring method described in previous reports $[25,29,30]$. Briefly, worms were classified as class " $\mathrm{A}$ " when they exhibited spontaneous movement or vigorous locomotion in response to prodding; class " $\mathrm{B}$ " worms were those that did not move unless prodded or appeared to have uncoordinated movement; and class " $\mathrm{C}$ " worms were those that moved only their head and/or tail in response to 
prodding. Dead worms were classified as class " $\mathrm{D}$ ". Two independent replicates were tested, and the merged data were statistically analyzed and presented.

\subsection{Stress Resistance Assays}

Worms were grown from an age of 3 days on mNGM plates containing OP50 or CBM 588 and then subjected to stress resistance assays. The bacterial infection was performed as previously shown [23]. Briefly, 7-day-old worms were transferred onto mNGM plate covered with $10 \mathrm{mg}$ of Staphylococcus aureus or Salmonella instead of OP50 or CBM 588, cultured at $25^{\circ} \mathrm{C}$, and the lifespan was determined. To assess thermal tolerance, 7 -day-old worms were incubated at $35{ }^{\circ} \mathrm{C}$ for $6 \mathrm{~h}$ and then scored for viability every hour. The following assays were performed as previously described [31]. 9-day-old worms were exposed to UV irradiation at $500 \mathrm{~J} / \mathrm{m}^{2}$ using a HL-2000 HybriLinker (UVP, Upland, CA, USA), incubated at $25^{\circ} \mathrm{C}$, and then scored for viability every day. For the oxidative stress assay, 9-day-old worms were transferred into M9 buffer containing $0.1 \%$ cholesterol $(5 \mathrm{mg} / \mathrm{mL}$ in ethanol) and $2 \mathrm{mM}$ hydrogen peroxide, and then scored for viability every hour. Similarly, for the heavy metal stress assay, 9-day-old worms were transferred to a 7-mM CuCl 2 solution. Survival was determined by touch-provoked movement. Worms were scored as dead when they failed to respond to repeated touching with a worm picker. The assays were performed at least twice.

\subsection{RNA Sequencing}

Three-day-old worms were cultured for five days on mNGM plates covered with OP50 (control-fed group) or CBM 588 (CBM 588-fed group). Approximately 200 worms in each group were collected and soaked in RNAlater solution (Qiagen, Hilden, Germany). Total RNA was isolated using the RNeasy Lipid Tissue kit (Qiagen). RNA sequencing was performed by DNAFORM (Yokohama, Japan) as follows: mRNAs were purified using the Magnosphere ${ }^{\mathrm{TM}}$ UltraPure mRNA Purification Kit (Clontech, Mountain View, CA, USA). Then, libraries prepared using the SMARTer ${ }^{\circledR}$ Stranded Total RNA-Seq Kit (Clontech) were run on HiSeq as 150 bp paired-end (Illumina Inc., San Diego, CA, USA). The quality of the RNA-seq results was first assessed using FastQC (ver. 0.11.7). The raw reads were trimmed and quality-filtered using the Trim Galore! (ver. 0.4.4), Trimmomatic (ver. 0.36) [32] and cutadapt (ver. 1.16) software. Clean reads were aligned versus the N2 Caenorhabditis elegans reference genome ce11 (WBcel235.91) using STAR (ver. 2.6.1a) [33]. Mapping statistics was presented in Table S1. After the read count of gene features was performed with the featureCounts tool (ver. 1.6.1) [34], a quantitative differential expression analysis between conditions was performed using DESeq (ver. 1.30.0) [35] to compare the control-fed and CBM 588-fed groups (see Table S2). Genes with I Log $2 \mathrm{FC} \mathrm{I}$ $\geq 2$ and $p$-value $<0.05$ (Tables S3 and S4) were subjected to the enrichment analysis based on the Gene Ontology (GO) category for Biological Process (BP), Molecular Function (MF), and Cellular Component (CC) using clusterProfiler (ver. 3.6.0) [36] (Tables S5 and S6). The raw and processed data files were deposited at Gene Expression Omnibus (GEO) (GSE123163).

\subsection{Reverse Transcription and Real-Time PCR}

Genomic DNA was removed and cDNA was synthesized using the QuantiTect Reverse Transcription Kit (Qiagen). Real-time PCR (quantitative PCR) was performed with a StepOnePlus Real-Time PCR system (Thermo Fisher Scientific, Waltham, MA, USA) using FastStart Universal SYBR Green Master (ROX) (Roche Diagnostics, Mannheim, Germany) with the following parameters: $95^{\circ} \mathrm{C}$ for $10 \mathrm{~min}$, and 40 cycles of $95^{\circ} \mathrm{C}$ for $15 \mathrm{~s}$ and $60{ }^{\circ} \mathrm{C}$ for $1 \mathrm{~min}$. Samples from three biological replicates were analyzed. Relative mRNA was determined with the $\Delta \Delta \mathrm{Ct}$ method [37] using expression of the $c y c-1$ or $t b a-1$ gene. The primers used for real-time PCR were as follows: $c y c-1$, (F: Forward) 5'-CGTGGTTCAAGGATCTAAACG-3' and (R: Reverse) 5'-ACCGAGTTCTCCAAAGCGTA-3'; $t b a-1$, (F) 5'-TCAACACTGCCATCGCCGCC-3' and (R) 5'-TCCAAGCGAGACCAGGCTTCAG-3'; gsto-1, (F) 5'-AGCTGCTAGCGGACAGGTTA-3' and (R) 5'-CGCTGCTTTTCATCTTTCAA-3'; and gsto-2, (F) 5'-TTGAACTTGCAAAAGCATATGATAC-3' and (R) 5'-TGAGATAATCGACAAATCCTGGT-3'; dod-22, 
(F) 5'-CTTCTTCGTCAAACCGCAAT-3' and (R) 5'-TGTGAGAGACTTCCGATGTAGG-3'; dod-23, (F) 5'-GATTCAGTGCGCTGTACCATAC-3' and (R) 5'-GTAGGCTTCCATCTTTGAGAGC-3'; dod-24, (F) 5'-CTTCTTCGTCAAACCGCAAT-3' and (R) 5'-TGTGAGAGACTTCCGATGTAGG-3'.

\section{Results and Discussion}

\subsection{CBM 588 Prolonged the Longevity of C. elegans}

To examine the effect of $C$. butyricum MIYAIRI 588 (CBM 588) on the C. elegans lifespan, worms were fed CBM 588 from an age of 3 days. As a result, the lifespan of the animals fed CBM 588 was significantly longer than that of the animals fed E. coli OP50 (Figure 1a). It is possible that the longevity that resulted from the feeding of CBM 588 may be due to dietary restriction (DR), which could be considerable if CBM 588 contains fewer calories or is too difficult for worms to digest. To test this possibility, CBM 588 was mixed with an equal volume of OP50 that was sufficient to avoid DR, and then, the animals were fed this mixture. This mixture of CBM 588 and OP50 also extended the lifespan compared with E. coli OP50 alone (Figure 1a). DR is known to decrease body weight and fertility in C. elegans $[38,39]$. The body size of the animals fed CBM 588 alone, but not the mixture of CBM 588 and OP50, was smaller compared with that of animals fed OP50 alone (Figure 1b). The brood size of the worms fed CBM 588 alone was not significantly different in comparison with the size of those fed OP50 alone (Figure 1c). In contrast, the brood size of the mixture-fed group was larger compared with the control-fed group (Figure 1c). Taken together, these findings indicate that CBM 588 prolonged the longevity of C. elegans at least partially in a DR-independent manner.

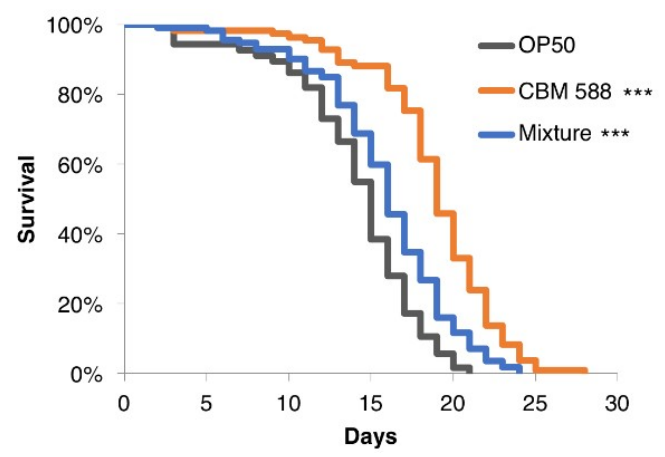

(a)

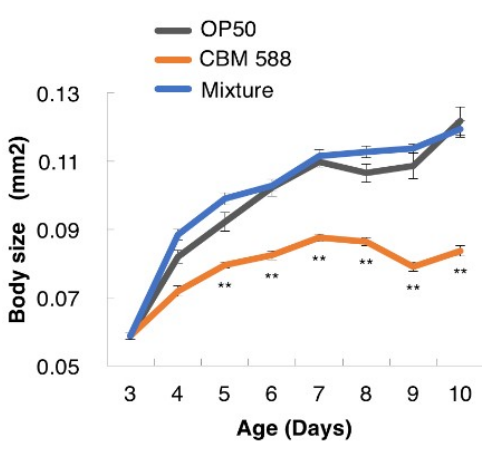

(b)

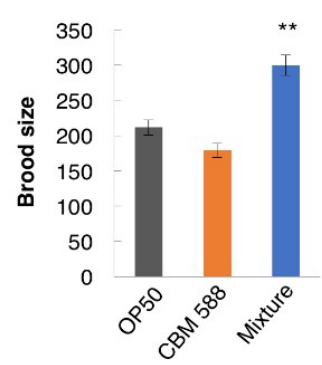

(c)

Figure 1. Clostridium butyricum MIYAIRI 588 (CBM 588) extended the lifespan of C. elegans. Survival curves (a), growth curves (b) and brood size (c) of animals fed OP50 (control), CBM 588, or a mixture of OP50 and CBM 588. (a) Worms were 3 days old on the nominal Day 0. OP50 (control)-fed group, $n=122$; CBM 588-fed group, $n=109$; mixture-fed group, $n=112$. Differences in survival were analyzed using the log-rank test. ${ }^{* * *} p<0.001$ vs. control-fed group. Detailed lifespan data and statistics are provided in Table S7. (b) The area of the worm's projection was measured. $n \geq 8$ for each assay. ** $p<0.01$ vs. control-fed group, ANOVA followed by post hoc Tukey-Kramer's test. (c) The brood size of worms fed CBM 588 alone was not significantly different in comparison with the size of those fed OP50 alone, and the brood size of the mixture-fed group was larger compared with the control-fed group. OP50 (control), $n=11$; CBM 588, $n=12$; mixture, $n=13$. ${ }^{* *} p<0.01$ vs. control-fed group, Statistical analysis was performed by ANOVA followed by post hoc Tukey-Kramer's test.

\subsection{UV-Killed CBM 588 Extended the Lifespan of C. elegans}

Although probiotics are defined as "live microorganisms that, when administered in adequate amounts, confer a health benefit on the host" by the Food and Agriculture Organization of the United Nations and the World Health Organization [1], growing evidence has shown that not only living bacteria but also dead bacteria can exert probiotic effects [40,41]. In addition, Hayashi et al. showed that heat-killed CBM 588 induced IL-10 production by macrophages [6]. We investigated whether 
UV-killed CBM 588 prolonged the lifespan of C. elegans. Notably, the lifespan of worms fed UV-killed CBM 588 was increased compared with UV-killed OP50 (Figure 2). Therefore, not only living CBM 588 but also dead CBM 588 exerted longevity effects in C. elegans.

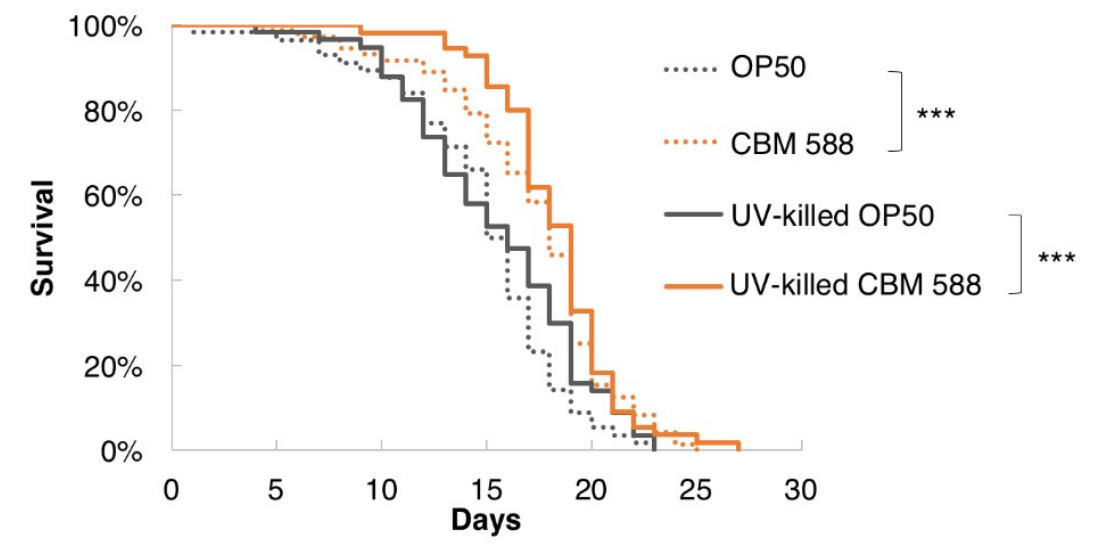

Figure 2. UV-killed CBM 588 prolonged the longevity of C. elegans. Survival curves of animals fed UV-killed bacteria. The lifespan of worms fed UV-killed CBM 588 was significantly increased compared with those fed UV-killed OP50. OP50, $n=56$; CBM 588, $n=72$; UV-killed OP50, $n=57$; UV-killed CBM $588, n=55 .{ }^{* * *} p<0.001, \log$-rank test. Detailed lifespan data and statistics are provided in Table S7.

\subsection{CBM588 Improved Locomotion during Aging in C. elegans}

To determine whether CBM 588 improved the decline of locomotion during aging, the motility of worms was scored. The scores of animals fed CBM 588 were higher than those of animals fed OP50 at every age tested (Figure 3a,b). The percentage of worms in class "A," wherein animals exhibited spontaneous movement or vigorous locomotion in response to prodding, was significantly increased in the CBM 588-fed group (Figure 3c). These results support the hypothesis that CBM 588 not only extends the lifespan of $C$. elegans but also improves health.

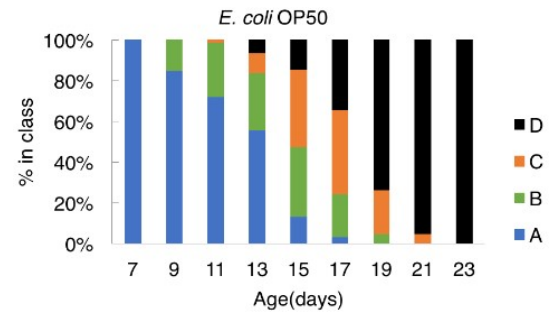

(a)

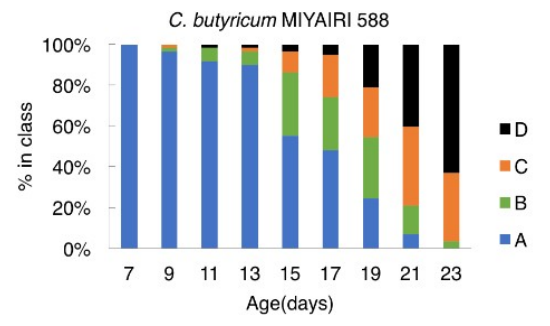

(b)

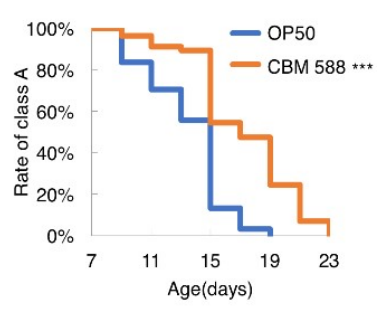

(c)

Figure 3. CBM588 improved locomotion during aging in C. elegans. (a,b) Locomotory activity of C. elegans fed OP (a) or CBM 588 (b). Animals were grouped into the following four classes based on their locomotion: class A, robust, coordinated sinusoidal locomotion (blue bars); class B, uncoordinated and/or sluggish movement (green bars); class C, no forward or backward movement, but head movements or shuddering in response to prodding (orange bars); and class D, dead animals (black bars). The frequency of each class at the indicated time point is indicated. $n \geq 57$ for each assay. (c) The

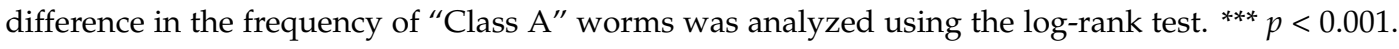
OP50, $n=61$, CBM 588, $n=57$.

\subsection{Genes That Were Regulated by the CBM 588 Feeding}

To characterize the genes that were regulated by feeding with CBM 588, RNA sequencing was performed (Tables S1 and S2). A total of 88 genes were shown to be increased in the CBM 588-fed group in comparison with the control-fed group with $\log _{2} \mathrm{FC} \geq 2$ and $p<0.05$ (Table S3). A total of 459 genes were decreased in the CBM 588-fed group with $\log _{2} \mathrm{FC} \leq 2$ and $p<0.05$ (Table S4). Significantly 
enriched Gene Ontology (GO) in the CBM 588-fed group is listed in Table S5 for upregulated genes and in Table S6 for downregulated genes. Within the upregulated genes, the top-ranked GO term for molecular function (MF) was "protein heterodimerization activity" that includes genes encoding for histone components. Because the second-ranked "structural constituent of cuticle" was also significant in the downregulated genes, this GO term was not specifically enriched in the upregulated genes. The expression of genes encoding for glucuronosyltransferases, endopeptidases and heme-binding proteins was also induced. cpr-5, an endopeptidase gene upregulated by CBM 588, has been reported to be induced by Lactobacillus acidophilus NCFM in a pmk-1/p38 MAPK-dependent manner [24]. Notably, "glutathione transferase activity" was significantly enriched in genes that were upregulated by CBM 588. The Omega class glutathione transferase gene gsto-1 has been reported to play key roles in counteracting environmental stress [42], and gsto-2 is a paralog of gsto-1. Fold-changes of the gsto-1 and gsto-2 gene were 45.5 and 5.3, respectively (Table S2). Using real-time PCR, we confirmed that the mRNA expression of gsto-1 and gsto-2 was increased in the CBM-fed group compared with the control-fed group (fold-change: gsto-1, $39.3 \pm 7.1$ (S.D.); gsto-2, $2.0 \pm 1.1$ ) (Figure 4a and Figure S1). Meanwhile, the top-ranked GO term for biological process (BP) in the downregulated genes was "defense response" (Table S6). Intriguingly, dod (downstream of DAF-16) genes such as dod-22 and dod-24, were found in the "defense response" category (Table S6). Tepper et al. has reported class I (upregulated) and class II (downregulated) DAF-16-responsible genes extracted from a meta-analysis, and dod-23, dod-22, and dod-24 were the very top 3-ranked genes in class II [43]. The dod-22, dod-23, and dod-24 genes were all decreased in the CBM 588-fed group in RNA sequencing (fold-change: $0.029,0.306$, and 0.002, respectively) (Table S2), and also in real-time PCR (0.0029 \pm 0.0002 (S.D.), $0.460 \pm 0.058,0.0042 \pm 0.0012$, respectively) (Figure $4 \mathrm{~b}$ and Figure S1). In contrast to class II, none of the class I genes listed in Table 1 of the Tepper's report [43], was found in Table S3. These results suggest that the DAF-16-dependent class II pathway but not class I, may be modulated by feeding with CBM 588.

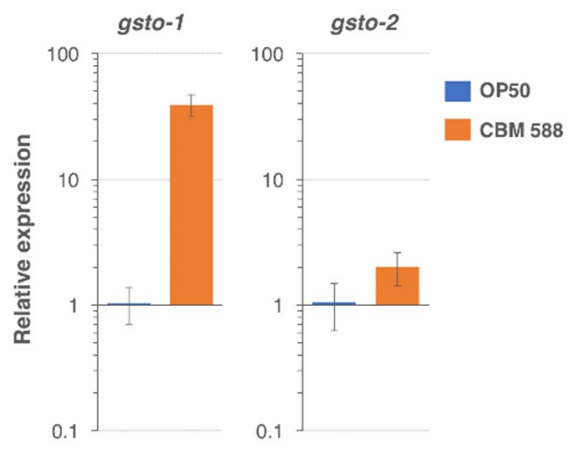

(a)

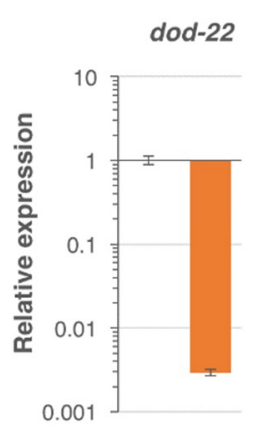

0.00

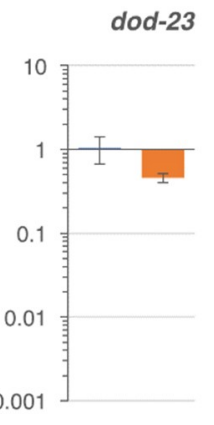

(b)

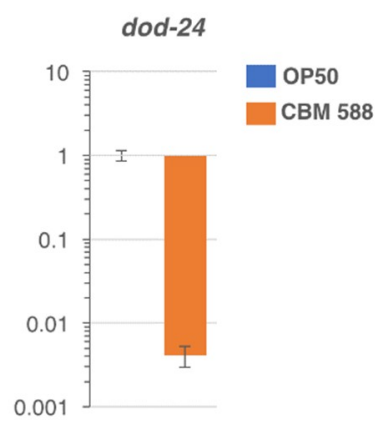

Figure 4. Relative gene expression (determined using real-time PCR) in the animals fed CBM 588 in comparison with control (OP50). (a) The gsto-1 and gsto-2 expression was upregulated by the CBM 588 feeding. (b) The expression of dod-22, dod-23, dod-24 was downregulated by CBM 588. Error bars indicate S.D. $n=3$ biological replicates.

\subsection{CBM 588 Increased Resistance to Biological Stresses in C. elegans}

Salmonella spp., which are Gram-negative bacteria, and Staphylococcus aureus, which are Gram-positive bacteria, shorten the lifespan of worms [23,44-46]. We found that probiotic bifidobacteria and lactobacilli could make C. elegans resilient to Salmonella infection [23]. Afterward, Kim et al. reported that the L. acidophilus strain NCFM specifically enhances Gram-positive immune responses; conditioning with $L$. acidophilus NCFM significantly prolonged the survival of nematodes exposed to the Gram-positive pathogens Enterococcus faecalis and Staphylococcus aureus, but this strain had a minimal effect on Gram-negative infection with Pseudomonas aeruginosa or Salmonella enterica [24]. To investigate the effect of CBM 588 on the biological stresses caused by pathogenic bacterial infection, 
7-day-old worms were exposed to Salmonella enterica or Staphylococcus aureus. Nematodes fed CBM 588 lived longer after being infected with Salmonella enterica or Staphylococcus aureus compared with worms fed OP50 (Figure 5a,b). In contrast to the results obtained with NCFM, worms fed CBM 588 exhibited increased resistance to both Gram-positive and -negative bacteria, Staphylococcus aureus and Salmonella enterica.

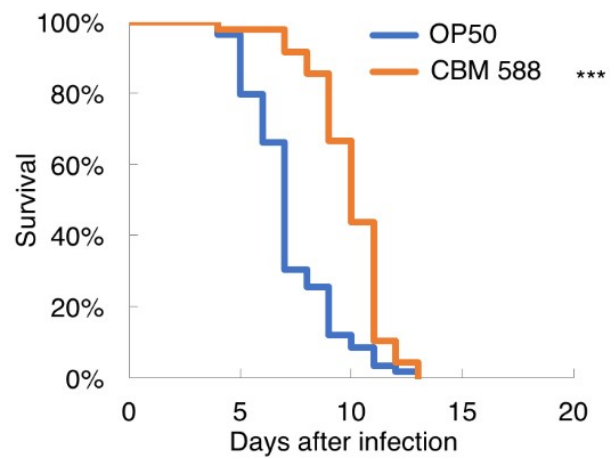

(a)

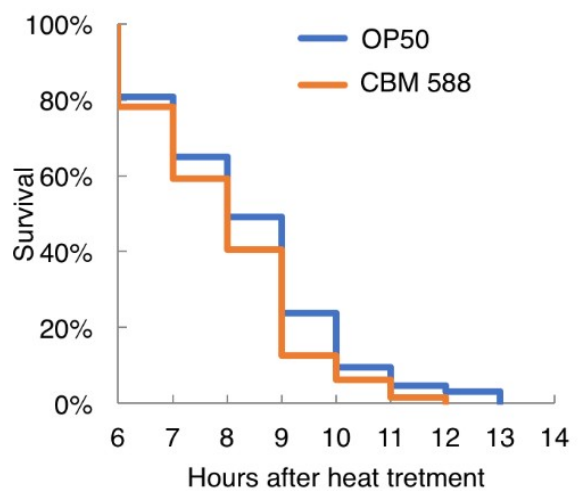

(c)

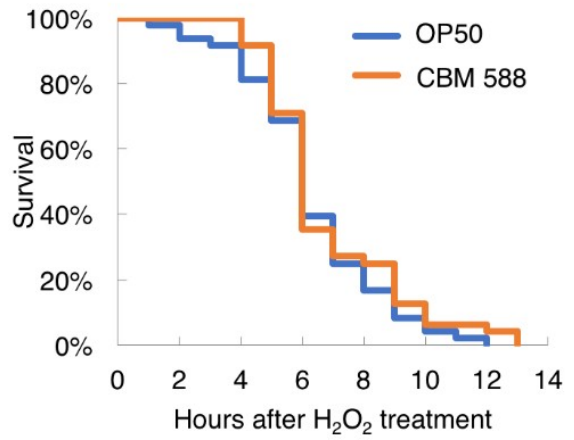

(e)

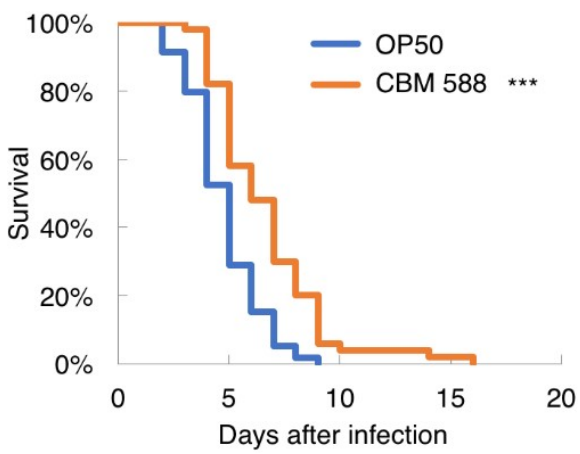

(b)

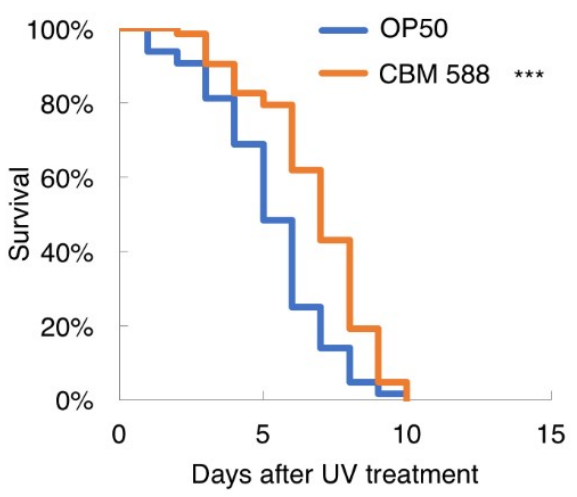

(d)

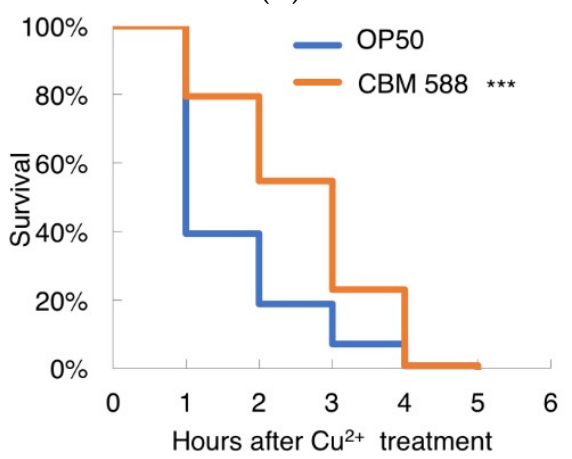

(f)

Figure 5. Susceptibility of CBM 588-fed worms to stresses. (a,b) Survival of $C$. elegans infected with pathogenic bacteria, Salmonella enterica (a) or Staphylococcus aureus (b). (a) OP50-fed group, $n=59$; CBM 588-fed group, $n=48$. (b) OP50-fed group, $n=59$; CBM 588-fed group, $n=50$. (c-f) Susceptibility of worms fed CBM 588 to heat stress (c), UV irradiation (d), hydrogen peroxide (e) and $\mathrm{Cu}^{2+}$ (f). (c) OP50, $n=64 ;$ CBM 588, $n=63$. (d) OP50, $n=64 ;$ CBM 588, $n=63$. (e) OP50, $n=48 ;$ CBM 588, $n=48$. (f) OP50, $n=63 ;$ CBM 588, $n=62$. Animals were fed OP50 (control) or CBM 588 from an age of 3 days and then exposed to the individual stresses at the ages described in the Materials and methods. Worms were 7 days old $(\mathbf{a}-\mathbf{c})$ or 9 days old $(\mathbf{d}-\mathbf{f})$ on the graph's nominal Day 0 . The survival curves were compared with those of worms fed OP50. ${ }^{* * *}$ Statistical significance at $p<0.001, \log$-rank test. 


\subsection{CBM 588 Increased Resistance to UV Irradiation and $\mathrm{Cu}^{2+}$ in C. elegans}

We next examined whether CBM 588 increased resistance to physical and chemical stressors. CBM 588 did not prolong the survival time of worms subjected to heat stress (Figure $5 \mathrm{c}$ ) but did increase the survival time of worms subjected to UV irradiation (Figure $5 \mathrm{~d}$ ). When the animals were exposed to hydrogen peroxide, which is a known source of active oxygen, the survival rates of OP-fed and CBM 588-fed worms were not significantly different (Figure 5e). In contrast, the CBM 588-fed animals lived longer compared to the OP50-fed animals in the presence of $\mathrm{Cu}^{2+}$ (Figure 5f). Transgenic animals overexpressing GSTO-1 were hyperresistant to oxidative stress, and silencing the expression of GSTO-1 by RNAi resulted in worms with an increased sensitivity to oxidants and heat shock [42]. Because CBM 588 conferred resistance to UV irradiation and $\mathrm{Cu}^{2+}$ but not to heat or $\mathrm{H}_{2} \mathrm{O}_{2}$ oxidative stress, upregulation of GSTO-1 is unlikely to be the underlying mechanism through which CBM 588 increases stress resistance.

\subsection{CBM 588 Could Extend the Lifespan of Worms through Regulation of the IIS Pathway and the Nrf2 Transcription Factor}

The insulin/IGF-1-like signaling (IIS) pathway plays a central role in stress resistance and longevity. In C. elegans, IIS is initiated by the binding of insulin-like peptides to the receptor DAF-2, which eventually leads to the activation of downstream IIS kinases, which phosphorylate and inhibit the FOXO transcription factor DAF-16 [47-50]. Moreover, transcriptional profiling using RNA sequencing implicated the involvement of the DAF-16-dependent class II pathway. To investigate whether the IIS pathway is involved in the prolongevity effects of CBM 588, the lifespans of the C. elegans loss-of-function mutants $d a f-2$ and $d a f-16$ were measured. CBM 588 failed to prolong the lifespan of strains harboring individual mutations in daf-2 or daf-16 (Figure 6). SKN-1 (an ortholog of mammalian Nrf2) is one of the key factors that regulates the stress responses and longevity of C. elegans [51,52]. Notably, CBM 588 failed to extend the lifespan of the $s k n-1$ mutants (Figure 6). These results suggest that both DAF-16 and SKN-1 may be required for the CBM 588-mediated lifespan extension. Because SKN-1 has been shown to be directly suppressed by DAF-2 [53], DAF-2 signaling via SKN-1 activity may also regulate the lifespan of worms fed CBM 588. The p38 MAPK pathway activates SKN-1 in several contexts, including pathogen infection and stress responses [54-56], raising the possibility that SKN-1 activation via the p38 MAPK pathway contributes to the prolongevity of animals fed CBM 588. However, we could not determine whether $p m k-1 / \mathrm{p} 38$ MAPK is required for the prolonged lifespan caused by CBM 588 because the pmk-1 mutation caused severe egg-laying defects when these mutants were fed CBM 588 (data not shown). Further analyses will be necessary to elucidate the role of the p38 MAPK pathway in the increased lifespan resulting from CBM 588. The long-lived mutants daf-2 and age-1 exhibited increased resistance to heat [57], oxidants [58,59], UV [60], and metal stress [61], indicating that longevity and stress resistance are closely related. Based on our results from the CBM 588-mediated lifespan extension, suppression of DAF-2 may partially mediate the increased resistance of animals fed CBM 588. However, the possibility that the impact of CBM 588 on longevity was insufficient to further extend the lifespan of the long-lived daf-2 mutants, cannot be ruled out. In addition, the experiments using mutants that were not outcrossed with our own N2 strain, require caution in the interpretation of these results, because it has been reported that the impact of simply a different N2 strain on lifespan can be huge [62]. 


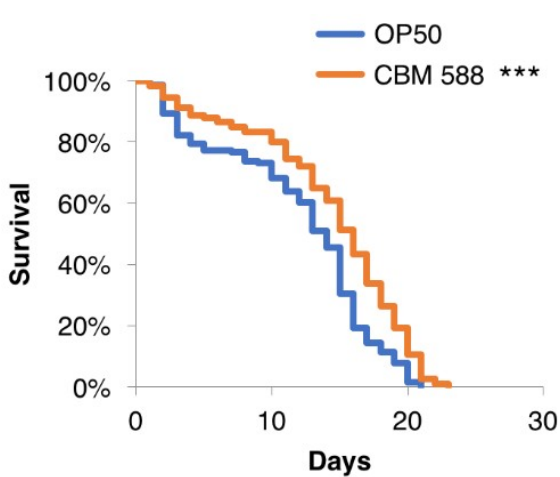

(a)

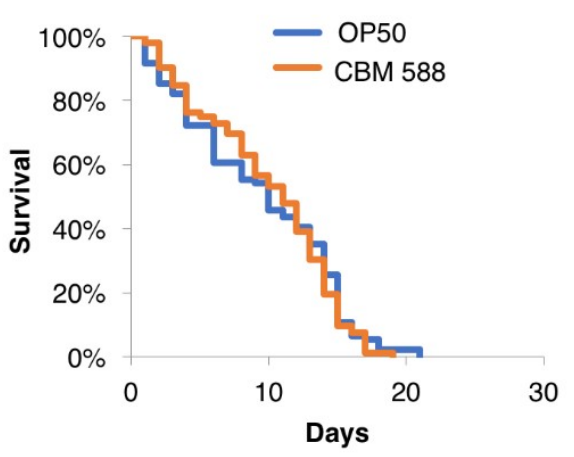

(c)

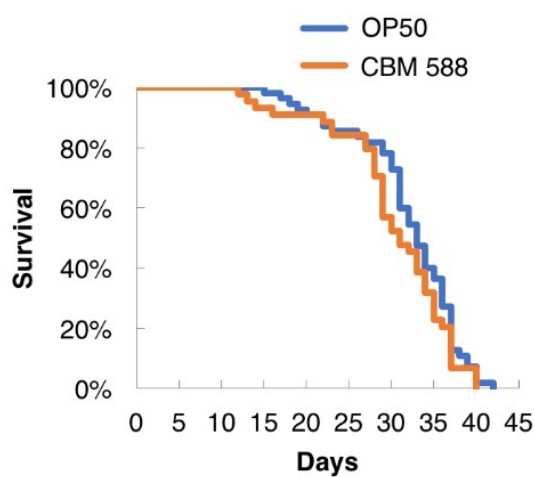

(b)

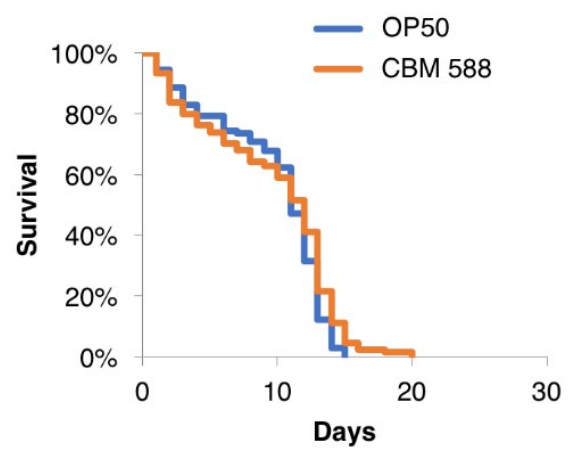

(d)

Figure 6. Effects of CBM 588 on the lifespan of C. elegans loss-of-function mutants. Survival curves of the wild-type animals (a) and the daf-2 (b), daf-16 (c), and skn-1 mutants (d). CBM 588 failed to extend the lifespan of these mutants. The survival curves were compared with those of worms fed OP50. *** Statistical significance at $p<0.001, \log$-rank test. Detailed lifespan data and statistics are provided in Table S7.

\section{Conclusions}

In the present study, we demonstrated for the first time that CBM 588 extends the lifespan of C. elegans. The involved mechanisms seemed to depend on the DAF-16/FOXO and the SKN-1/Nrf2 transcription factors. Our transcriptional profiling comparing CBM 588-fed and control-fed animals suggested that DAF-16-dependent class II genes were regulated by CBM 588. Taken together with the fact that reduced IIS extends the lifespan of C. elegans by upregulating stress response (class I) and downregulating other (class II) genes in a DAF-16-dependent manner [43], CBM 588 may exert its prolongevity through modulating of the DAF-16 class II pathway. Because DAF-16/FOXO is well-conserved transcription factor that regulate longevity among animal species including humans, it would be worth studying the DAF-16-dependent mechanisms that govern the effects of the probiotic CBM 588.

Supplementary Materials: The following are available online at http://www.mdpi.com/2072-6643/10/12/ 1921/s1, Figure S1: Gene expression (real-time PCR) in the animals fed OP50 or CBM 588. Table S1: Mapping statistics. Table S2: Differential expression analysis using DESeq (CBM 588 vs. OP50 (control)). Table S3: Genes upregulated in CBM-fed animals. Table S4: Genes downregulated in CBM-fed animals. Table S5: Gene ontology (GO) enrichment for genes upregulated by CBM 588. Table S6: Gene ontology (GO) enrichment for genes downregulated by CBM 588. Table S7: Summary of lifespan experiments.

Author Contributions: Investigation, M.K., Y.H., S.S.; writing-original draft preparation, E.K.-N., Y.N.; supervision, E.K.-N., Y.N.; project administration, E.K.-N., Y.N.

Funding: This research received no external funding.

Acknowledgments: We thank the Caenorhabditis Genetics Center (University of Minnesota, Minneapolis, MN; supported by the National Institutes of Health-National Center for Research Resources) for providing the C. elegans strains, and Miyarisan Pharmaceutical for providing C. butyricum MIYAIRI 588. 
Conflicts of Interest: The authors declare no conflict of interest.

\section{References}

1. Sanders, M.E. Probiotics: Definition, sources, selection, and uses. Clin. Infect. Dis. 2008, 46 (Suppl. 2), S58-S61, discussion S144-S151. [CrossRef] [PubMed]

2. Cassir, N.; Benamar, S.; La Scola, B. Clostridium butyricum: From beneficial to a new emerging pathogen. Clin. Microbiol. Infect. 2016, 22, 37-45. [CrossRef] [PubMed]

3. Ferraris, L.; Butel, M.J.; Aires, J. Antimicrobial susceptibility and resistance determinants of Clostridium butyricum isolates from preterm infants. Int. J. Antimicrob. Agents 2010, 36, 420-423. [CrossRef] [PubMed]

4. Sato, R.; Tanaka, M. Intestinal distribution and intraluminal localization of orally administered Clostridium butyricum in rats. Microbiol. Immunol. 1997, 41, 665-671. [CrossRef] [PubMed]

5. Seki, H.; Shiohara, M.; Matsumura, T.; Miyagawa, N.; Tanaka, M.; Komiyama, A.; Kurata, S. Prevention of antibiotic-associated diarrhea in children by Clostridium butyricum MIYAIRI. Pediatr. Int. 2003, 45, 86-90. [CrossRef] [PubMed]

6. Hayashi, A.; Sato, T.; Kamada, N.; Mikami, Y.; Matsuoka, K.; Hisamatsu, T.; Hibi, T.; Roers, A.; Yagita, H.; Ohteki, T.; et al. A single strain of Clostridium butyricum induces intestinal IL-10-producing macrophages to suppress acute experimental colitis in mice. Cell Host Microbe 2013, 13, 711-722. [CrossRef] [PubMed]

7. Kashiwagi, I.; Morita, R.; Schichita, T.; Komai, K.; Saeki, K.; Matsumoto, M.; Takeda, K.; Nomura, M.; Hayashi, A.; Kanai, T.; et al. Smad 2 and Smad3 Inversely Regulate TGF- $\beta$ Autoinduction in Clostridium butyricum-Activated Dendritic Cells. Immunity 2015, 43, 65-79. [CrossRef]

8. Seo, M.; Inoue, I.; Tanaka, M.; Matsuda, N.; Nakano, T.; Awata, T.; Katayama, S.; Alpers, D.H.; Komoda, T. Clostridium butyricum MIYAIRI 588 improves high-fat diet-induced non-alcoholic fatty liver disease in rats. Dig. Dis. Sci. 2013, 58, 3534-3544. [CrossRef]

9. Woo, T.D.; Oka, K.; Takahashi, M.; Hojo, F.; Osaki, T.; Hanawa, T.; Kurata, S.; Yonezawa, H.; Kamiya, S. Inhibition of the cytotoxic effect of Clostridium difficile in vitro by Clostridium butyricum MIYAIRI 588 strain. J. Med. Microbiol. 2011, 60, 1617-1625. [CrossRef]

10. Takahashi, M.; Taguchi, H.; Yamaguchi, H.; Osaki, T.; Komatsu, A.; Kamiya, S. The effect of probiotic treatment with Clostridium butyricum on enterohemorrhagic Escherichia coli O157:H7 infection in mice. FEMS Immunol. Med. Microbiol. 2004, 41, 219-226. [CrossRef]

11. Wang, F.Y.; Liu, J.M.; Luo, H.H.; Liu, A.H.; Jiang, Y. Potential protective effects of Clostridium butyricum on experimental gastric ulcers in mice. World J. Gastroenterol. 2015, 21, 8340-8351. [CrossRef] [PubMed]

12. Finch, C.E.; Ruvkun, G. The genetics of aging. Annu. Rev. Genom. Hum. Genet. 2001, 2, 435-462. [CrossRef]

13. Hodgkin, J.; Doniach, T. Natural variation and copulatory plug formation in Caenorhabditis elegans. Genetics 1997, 146, 149-164. [PubMed]

14. Barrière, A.; Félix, M.A. High local genetic diversity and low outcrossing rate in Caenorhabditis elegans natural populations. Curr. Biol. 2005, 15, 1176-1184. [CrossRef] [PubMed]

15. Barrière, A.; Félix, M.A. Temporal dynamics and linkage disequilibrium in natural Caenorhabditis elegans populations. Genetics 2007, 176, 999-1011. [CrossRef] [PubMed]

16. Félix, M.A.; Duveau, F. Population dynamics and habitat sharing of natural populations of Caenorhabditis elegans and C. briggsae. BMC Biol. 2012, 10, 59. [CrossRef] [PubMed]

17. Frézal, L.; Félix, M.A. C. elegans outside the Petri dish. eLife 2015, 4. [CrossRef] [PubMed]

18. Berg, M.; Stenuit, B.; Ho, J.; Wang, A.; Parke, C.; Knight, M.; Alvarez-Cohen, L.; Shapira, M. Assembly of the Caenorhabditis elegans gut microbiota from diverse soil microbial environments. ISME J. 2016, 10, 1998-2009. [CrossRef]

19. Dirksen, P.; Marsh, S.A.; Braker, I.; Heitland, N.; Wagner, S.; Nakad, R.; Mader, S.; Petersen, C.; Kowallik, V.; Rosenstiel, P.; et al. The native microbiome of the nematode Caenorhabditis elegans: Gateway to a new host-microbiome model. BMC Biol. 2016, 14, 38. [CrossRef]

20. Samuel, B.S.; Rowedder, H.; Braendle, C.; Félix, M.A.; Ruvkun, G. Caenorhabditis elegans responses to bacteria from its natural habitats. Proc. Natl. Acad. Sci. USA 2016, 113, E3941-E3949. [CrossRef]

21. Zhang, F.; Berg, M.; Dierking, K.; Félix, M.A.; Shapira, M.; Samuel, B.S.; Schulenburg, H. Caenorhabditis elegans as a Model for Microbiome Research. Front. Microbiol. 2017, 8, 485. [CrossRef] [PubMed]

22. Brenner, S. The genetics of Caenorhabditis elegans. Genetics 1974, 77, 71-94. [PubMed] 
23. Ikeda, T.; Yasui, C.; Hoshino, K.; Arikawa, K.; Nishikawa, Y. Influence of lactic acid bacteria on longevity of Caenorhabditis elegans and host defense against salmonella enterica serovar enteritidis. Appl. Environ. Microbiol. 2007, 73, 6404-6409. [CrossRef] [PubMed]

24. Kim, Y.; Mylonakis, E. Caenorhabditis elegans immune conditioning with the probiotic bacterium Lactobacillus acidophilus strain NCFM enhances gram-positive immune responses. Infect. Immun. 2012, 80, 2500-2508. [CrossRef] [PubMed]

25. Komura, T.; Ikeda, T.; Yasui, C.; Saeki, S.; Nishikawa, Y. Mechanism underlying prolongevity induced by bifidobacteria in Caenorhabditis elegans. Biogerontology 2013, 14, 73-87. [CrossRef] [PubMed]

26. Cabreiro, F.; Gems, D. Worms need microbes too: Microbiota, health and aging in Caenorhabditis elegans. EMBO Mol. Med. 2013, 5, 1300-1310. [CrossRef] [PubMed]

27. Clark, L.C.; Hodgkin, J. Commensals, probiotics and pathogens in the Caenorhabditis elegans model. Cell. Microbiol. 2014, 16, 27-38. [CrossRef] [PubMed]

28. Wu, D.; Rea, S.L.; Yashin, A.I.; Johnson, T.E. Visualizing hidden heterogeneity in isogenic populations of C. elegans. Exp. Gerontol. 2006, 41, 261-270. [CrossRef] [PubMed]

29. Gruber, J.; Ng, L.F.; Fong, S.; Wong, Y.T.; Koh, S.A.; Chen, C.B.; Shui, G.; Cheong, W.F.; Schaffer, S.; Wenk, M.R.; et al. Mitochondrial changes in ageing Caenorhabditis elegans-What do we learn from superoxide dismutase knockouts? PLoS ONE 2011, 6, e19444. [CrossRef]

30. Hosono, R.; Sato, Y.; Aizawa, S.I.; Mitsui, Y. Age-dependent changes in mobility and separation of the nematode Caenorhabditis elegans. Exp. Gerontol. 1980, 15, 285-289. [CrossRef]

31. Yaguchi, Y.; Komura, T.; Kashima, N.; Tamura, M.; Kage-Nakadai, E.; Saeki, S.; Terao, K.; Nishikawa, Y. Influence of oral supplementation with sesamin on longevity of Caenorhabditis elegans and the host defense. Eur. J. Nutr. 2014, 53, 1659-1668. [CrossRef] [PubMed]

32. Bolger, A.M.; Lohse, M.; Usadel, B. Trimmomatic: A flexible trimmer for Illumina sequence data. Bioinformatics 2014, 30, 2114-2120. [CrossRef] [PubMed]

33. Dobin, A.; Davis, C.A.; Schlesinger, F.; Drenkow, J.; Zaleski, C.; Jha, S.; Batut, P.; Chaisson, M.; Gingeras, T.R. STAR: Ultrafast universal RNA-seq aligner. Bioinformatics 2013, 29, 15-21. [CrossRef] [PubMed]

34. Liao, Y.; Smyth, G.K.; Shi, W. featureCounts: An efficient general purpose program for assigning sequence reads to genomic features. Bioinformatics 2014, 30, 923-930. [CrossRef]

35. Anders, S.; Huber, W. Differential expression analysis for sequence count data. Genome Biol. 2010, 11, R106. [CrossRef]

36. Yu, G.; Wang, L.G.; Han, Y.; He, Q.Y. clusterProfiler: An R package for comparing biological themes among gene clusters. OMICS 2012, 16, 284-287. [CrossRef]

37. Livak, K.J.; Schmittgen, T.D. Analysis of relative gene expression data using real-time quantitative PCR and the 2(-Delta Delta C(T)) Method. Methods 2001, 25, 402-408. [CrossRef]

38. Klass, M.R. Aging in the nematode Caenorhabditis elegans: Major biological and environmental factors influencing life span. Mech. Ageing Dev. 1977, 6, 413-429. [CrossRef]

39. Tain, L.S.; Lozano, E.; Sáez, A.G.; Leroi, A.M. Dietary regulation of hypodermal polyploidization in C. elegans. BMC Dev. Biol. 2008, 8, 28. [CrossRef]

40. Tanaka, A.; Seki, M.; Yamahira, S.; Noguchi, H.; Kosai, K.; Toba, M.; Morinaga, Y.; Miyazaki, T.; Izumikawa, K.; Kakeya, H.; et al. Lactobacillus pentosus strain b240 suppresses pneumonia induced by Streptococcus pneumoniae in mice. Lett. Appl. Microbiol. 2011, 53, 35-43. [CrossRef]

41. Chen, M.F.; Weng, K.F.; Huang, S.Y.; Liu, Y.C.; Tseng, S.N.; Ojcius, D.M.; Shih, S.R. Pretreatment with a heat-killed probiotic modulates monocyte chemoattractant protein-1 and reduces the pathogenicity of influenza and enterovirus 71 infections. Mucosal Immunol. 2017, 10, 215-227. [CrossRef] [PubMed]

42. Burmeister, C.; Lüersen, K.; Heinick, A.; Hussein, A.; Domagalski, M.; Walter, R.D.; Liebau, E. Oxidative stress in Caenorhabditis elegans: Protective effects of the Omega class glutathione transferase (GSTO-1). FASEB J. 2008, 22, 343-354. [CrossRef] [PubMed]

43. Tepper, R.G.; Ashraf, J.; Kaletsky, R.; Kleemann, G.; Murphy, C.T.; Bussemaker, H.J. PQM-1 complements DAF-16 as a key transcriptional regulator of DAF-2-mediated development and longevity. Cell 2013, 154, 676-690. [CrossRef] [PubMed]

44. Aballay, A.; Yorgey, P.; Ausubel, F.M. Salmonella typhimurium proliferates and establishes a persistent infection in the intestine of Caenorhabditis elegans. Curr. Biol. 2000, 10, 1539-1542. [CrossRef] 
45. Labrousse, A.; Chauvet, S.; Couillault, C.; Kurz, C.L.; Ewbank, J.J. Caenorhabditis elegans is a model host for Salmonella typhimurium. Curr. Biol. 2000, 10, 1543-1545. [CrossRef]

46. Sifri, C.D.; Begun, J.; Ausubel, F.M.; Calderwood, S.B. Caenorhabditis elegans as a model host for Staphylococcus aureus pathogenesis. Infect. Immun. 2003, 71, 2208-2217. [CrossRef] [PubMed]

47. Murphy, C.T.; McCarroll, S.A.; Bargmann, C.I.; Fraser, A.; Kamath, R.S.; Ahringer, J.; Li, H.; Kenyon, C. Genes that act downstream of DAF-16 to influence the lifespan of Caenorhabditis elegans. Nature 2003, 424, 277-283. [CrossRef] [PubMed]

48. Berman, J.R.; Kenyon, C. Germ-cell loss extends C. elegans life span through regulation of DAF-16 by kri-1 and lipophilic-hormone signaling. Cell 2006, 124, 1055-1068. [CrossRef]

49. Mukhopadhyay, A.; Oh, S.W.; Tissenbaum, H.A. Worming pathways to and from DAF-16/FOXO. Exp. Gerontol. 2006, 41, 928-934. [CrossRef]

50. Samuelson, A.V.; Carr, C.E.; Ruvkun, G. Gene activities that mediate increased life span of C. elegans insulin-like signaling mutants. Genes Dev. 2007, 21, 2976-2994. [CrossRef]

51. Park, S.K.; Tedesco, P.M.; Johnson, T.E. Oxidative stress and longevity in Caenorhabditis elegans as mediated by SKN-1. Aging Cell 2009, 8, 258-269. [CrossRef] [PubMed]

52. Blackwell, T.K.; Steinbaugh, M.J.; Hourihan, J.M.; Ewald, C.Y.; Isik, M. SKN-1/Nrf, stress responses, and aging in Caenorhabditis elegans. Free Radic. Biol. Med. 2015, 88, 290-301. [CrossRef] [PubMed]

53. Tullet, J.M.; Hertweck, M.; An, J.H.; Baker, J.; Hwang, J.Y.; Liu, S.; Oliveira, R.P.; Baumeister, R.; Blackwell, T.K. Direct inhibition of the longevity-promoting factor $\mathrm{SKN}-1$ by insulin-like signaling in C. elegans. Cell 2008, 132, 1025-1038. [CrossRef] [PubMed]

54. Inoue, H.; Hisamoto, N.; An, J.H.; Oliveira, R.P.; Nishida, E.; Blackwell, T.K.; Matsumoto, K. The C. elegans p38 MAPK pathway regulates nuclear localization of the transcription factor SKN-1 in oxidative stress response. Genes Dev. 2005, 19, 2278-2283. [CrossRef] [PubMed]

55. Hoeven, R.; McCallum, K.C.; Cruz, M.R.; Garsin, D.A. Ce-Duox1/BLI-3 generated reactive oxygen species trigger protective SKN-1 activity via p38 MAPK signaling during infection in C. elegans. PLoS Pathog. 2011, 7, e1002453. [CrossRef]

56. Papp, D.; Csermely, P.; Sóti, C. A role for SKN-1/Nrf in pathogen resistance and immunosenescence in Caenorhabditis elegans. PLoS Pathog. 2012, 8, e1002673. [CrossRef] [PubMed]

57. MacColl, G.S.; Quinton, R.; Bülow, H.E. Biology of KAL1 and its orthologs: Implications for X-linked Kallmann syndrome and the search for novel candidate genes. Front. Horm. Res. 2010, 39, 62-77. [CrossRef] [PubMed]

58. Larsen, P.L. Aging and resistance to oxidative damage in Caenorhabditis elegans. Proc. Natl. Acad. Sci. USA 1993, 90, 8905-8909. [CrossRef]

59. Honda, Y.; Honda, S. The daf-2 gene network for longevity regulates oxidative stress resistance and Mn-superoxide dismutase gene expression in Caenorhabditis elegans. FASEB J. 1999, 13, 1385-1393. [CrossRef]

60. Murakami, S.; Johnson, T.E. A genetic pathway conferring life extension and resistance to UV stress in Caenorhabditis elegans. Genetics 1996, 143, 1207-1218.

61. Barsyte, D.; Lovejoy, D.A.; Lithgow, G.J. Longevity and heavy metal resistance in daf-2 and age-1 long-lived mutants of Caenorhabditis elegans. FASEB J. 2001, 15, 627-634. [CrossRef] [PubMed]

62. Zhao, Y.; Long, L.; Xu, W.; Campbell, R.F.; Large, E.E.; Greene, J.S.; McGrath, P.T. Changes to social feeding behaviors are not sufficient for fitness gains of the. eLife 2018, 7. [CrossRef] [PubMed]

(C) 2018 by the authors. Licensee MDPI, Basel, Switzerland. This article is an open access article distributed under the terms and conditions of the Creative Commons Attribution (CC BY) license (http://creativecommons.org/licenses/by/4.0/). 